\title{
Comparison Study of the Use of Absorbable Materials as Internal Splints with Airway Silicone Splint and Absorbable Materials as Internal Splints Alone
}

\author{
So Young Ji, \\ Seung Soo Kim, \\ Ki Sung Park, \\ Bong Soo Baik \\ Department of Plastic and Reconstructive \\ Surgery, Dong Kang General Hospital, Ulsan, \\ Korea
}

No potential conflict of interest relevant to this article was reported.

\begin{abstract}
Background: Packing after closed reduction of nasal fracture causes uncomfortable nasal obstruction in patients. We packed the superior meatus with synthetic polyurethane foam (SPF) to support the nasal bone, and packed the middle nasal meatus with a nasal airway splint (NAS) and SPF. The aim of this article is prospectively to compare the subjective patient discomfort of SPF (Nasopore Forte plus) packing alone and SPF with NAS.

Methods: We compared the prospectively subjective patient discomfort of SPF packing alone (group A) and SPF with NAS (group B) via visual analog scale (VAS; 0 , no symptom; 100, most severe symptom).

Results: At first postoperative day group B showed significant lower scores in dry mouth, sleep disturbance, conversation difficulty. However at third postoperative day, VAS scores of each group had no statistically significant differences. Moreover at fifth postoperative day group A had statistically significant lower scores for nasal pain, dry mouth than the group B.

Conclusion: Combination method of using NAS and SPF have some advantage on the patient comfort from first postoperative day to third postoperative day.
\end{abstract}

Keywords: Nasal bone / Airway management / Splints

\section{INTRODUCTION}

Nasal bone fracture is the most common fracture in facial trau$\mathrm{ma}$, and general treatment for fracture is closed reduction, which requires an internal splint in the nasal cavity to stabilize the reduced bone fragments [1].

Because the functions of the internal splint include the stabilization of the bone segments, epistaxis control, prevention of synechia formation, and discharge absorption, the splint material is

Correspondence: Bong Soo Baik

Department of Plastic and Reconstructive Surgery, Dong Kang General Hospital, 239,

Taehwa-ro, Jung-gu, Ulsan 44455, Korea

E-mail: bsbaiksy@naver.com

Received September 2, 2016 / Revised December 14, 2016 / Accepted Dec December 14, 2016 important. Furthermore, nasal packing in the internal splint can cause painful side effects, including dyspnea, sore throat, and headache [1].

The use of synthetic polyurethane foam (SPF) as an absorbable packing material can be a reasonable substitute for the traditional non-absorbable material [2], and the usefulness of the airway silicone splint with vaseline gauze has been proved [3].

We developed a combination method of nasal bone support using SPF (Nasopore Forte plus, Polyganics Rosenburglaan, Groningen, The Netherlands) in the superior meatus, and a nasal airway splint (NAS, Boston Medical Products Inc., Westborough, MA, USA) (Fig. 1) in the middle meatus. The aim of this article is prospectively to compare the subjective patient discomfort of SPF 




Fig. 1. Nasal airway splint (Boston Medical Products Inc., Westborough, MA, USA).

packing alone and SPF with NAS.

\section{METHODS}

Only pure nasal bone fracture patients who did not exhibit any other fractures were included. This trial was conducted on $40 \mathrm{pa}-$ tients between 20 and 60 years old who underwent closed reduction of a nasal fracture from September 2014 to September 2015.

Twenty patients were randomized into a group (group A) who had nasal packing using SPF alone, and the remaining 20 patients (group B) had nasal packing with SPF and NAS. Fracture sites were identified in all patients through preoperative radiography and computed tomography (CT) scans.

The operation was performed under general anesthesia. Before nasal reduction, epinephrine-soaked gauzes were packed into both nostrils for 10 minutes to control epistaxis. After the fractured nasal bone and septum were reduced in situ, an internal splint of SPF was applied in the superior meatus, and $8 \mathrm{~cm}$ of SPF was packed in the middle meatus without manipulation. On group B NAS was inserted into the middle meatus. Trans-septal suture was done and SPF was also applied in the superior and middle meatus to support NAS (Fig. 2).

The nasal irrigation was started on the first postoperative day on both groups, NAS was removed on the tenth postoperative day. The external nasal splint (External Nasal Splint Singles, Medtronic Xomed Surgical Products, Jacksonville, FL, USA) was maintained for 1 month after surgery.

On group B, if nasal respiration decreased due to blood clot or
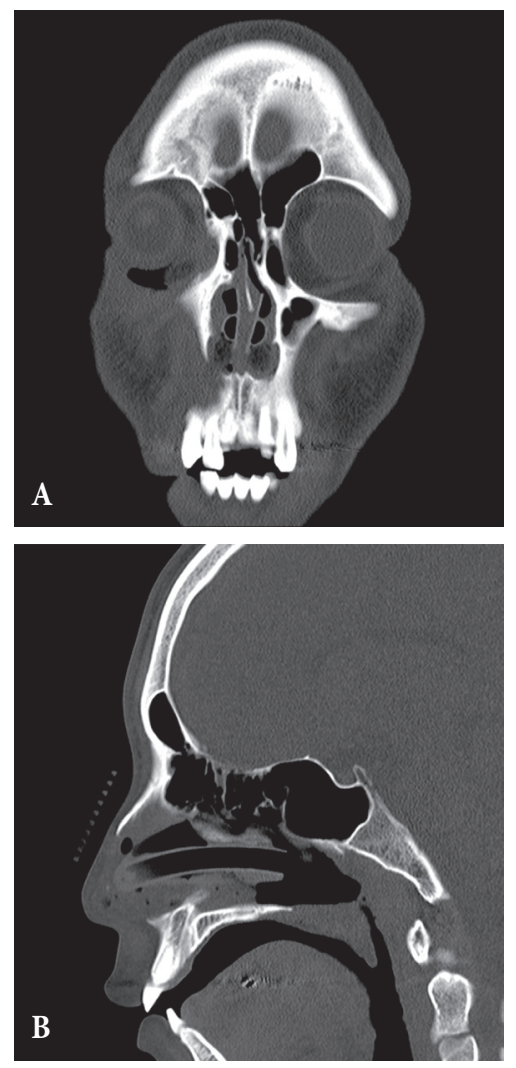

Fig. 2. Status of nasal airway splint in the computed tomography scan. (A) Coronal. (B) Sagittal.

intranasal discharge, the airway tube was sucked by applying negative pressure with a 10-Fr Nelaton tube during hospital days.

All patients completed a survey of nasal pain, dry mouth, sleep disturbance, swallowing difficulty, conversation, headache were assessed for five days after operation using the visual analog scale (VAS; 0, no symptom; 100, most severe symptom). For statistical analysis, SPSS ver. 18.0 (IBM, Armonk, NY, USA) was used. Measured values are expressed as averages and standard deviations. Group A and group B were compared through the Student's $t$-test and were considered significant when $p$-value was $<0.05$.

\section{RESULTS}

At first postoperative day, group B had statistically significant lower scores for dry mouth, sleep disturbance, conversation difficulty than group A $(p<0.001)$. The scores for nasal pain, swallowing difficulty, headache were lower in the group B, but the two 
groups had no statistically significant differences (Table 1, Fig. 3).

However at third postoperative day, VAS scores for dry mouth reversed to 14.8 in group A and 16.4 in the group B. But VAS scores of each group had no statistically significant differences (Table 2, Fig. 4). At fifth postoperative day group A had statistically significant lower scores for nasal pain, dry mouth than the group B $(p<0.001)$ (Table 3, Fig. 5).

\section{DISCUSSION}

After reduction of nasal bone fracture, an internal splint is necessary to support the reduced bone fragments. To date, nasal packing has been the only method for internal splinting. Because of

Table 1. Comparison of VAS scores on the first postoperation day

\begin{tabular}{lccc} 
Variable & Group A $(n=20)$ & Group B $(n=20)$ & $p$-value ${ }^{\text {a }}$ \\
\hline Nasal pain & $49.6 \pm 14.7$ & $44.6 \pm 15.1$ & 0.06 \\
Dry mouth & $67.8 \pm 17.2$ & $36.4 \pm 12.0$ & $<0.001^{*}$ \\
\hline Sleep disturbance & $68.9 \pm 17.4$ & $40.8 \pm 16.2$ & $<0.001^{*}$ \\
Swallowing difficulty & $69.6 \pm 16.2$ & $65.2 \pm 15.2$ & 0.11 \\
Conversation & $64.6 \pm 15.5$ & $32.3 \pm 11.8$ & $<0.001^{*}$ \\
Headache & $34.0 \pm 10.0$ & $33.0 \pm 10.10$ & 0.08 \\
\hline
\end{tabular}

VAS, visual analog scale.

a) Student's $t$-test; ${ }^{*} p$-value $<0.001$.

\section{First postoperation day}

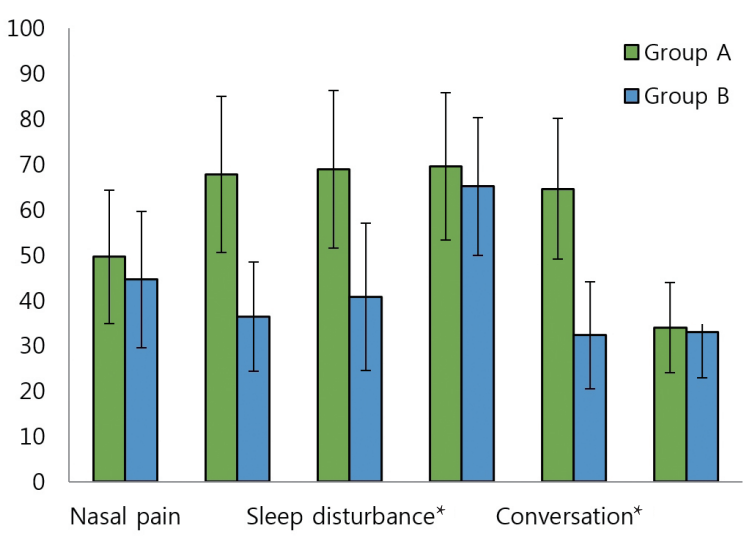

Fig. 3. Comparison of VAS scores on the first post operation day. Group B had statistically significant lower scores for dry mouth, sleep disturbance, conversation difficulty than group A $(p<0.001)$. The scores for nasal pain, swallowing difficulty, headache were lower in the group B, but the two groups had no statistically significant differences. VAS, visual analog scale. ${ }^{*} p$-value $<0.001$. the other roles of the nasal packing material, such as hemostasis, discharge absorption, and synechia prevention, intranasal packing is considered an essential procedure after closed reduction [1].

Thus, nasal packing is applied despite the nasal airway obstruction that can result in painful complications such as headache, dry mouth and cough.

Yi et al. [2] reported that SPF shows a similar hemostatic effect and absorption of discharge as a packing material as compared to the traditional material, polyvinyl alcohol sponge (merocel) and showed less complications related to packing removal. Kim et al. [3] reported decreased discomfort due to packing with maintaining nasal ventilation of the middle meatus by filling it with NAS. Choi et al. [4] reported one day packing had comparable postoperative outcome with reducing the patients' discomfort. We devised a method to minimize discomfort combined NAS and SPF

Table 2. Comparison of VAS scores on the third postoperation day

Variable Group A $(n=20)$ Group B $(n=20) \quad p$-value ${ }^{a)}$

\begin{tabular}{lccc} 
Nasal pain & $29.6 \pm 10.7$ & $24.6 \pm 12.1$ & 0.08 \\
\hline Dry mouth & $14.8 \pm 4.5$ & $16.4 \pm 5.0$ & 0.17 \\
Sleep disturbance & $10.5 \pm 4.0$ & $10.0 \pm 3.3$ & 0.37 \\
\hline Swallowing difficulty & $29.6 \pm 7.2$ & $25.2 \pm 8.2$ & 0.08 \\
\hline Conversation & $14.6 \pm 3.5$ & $12.3 \pm 4.8$ & 0.10 \\
\hline Headache & $14.0 \pm 4.1$ & $13.0 \pm 3.1$ & 0.12 \\
\hline
\end{tabular}

VAS, visual analog scale. a)Student'st-test.

\section{Third postoperation day}

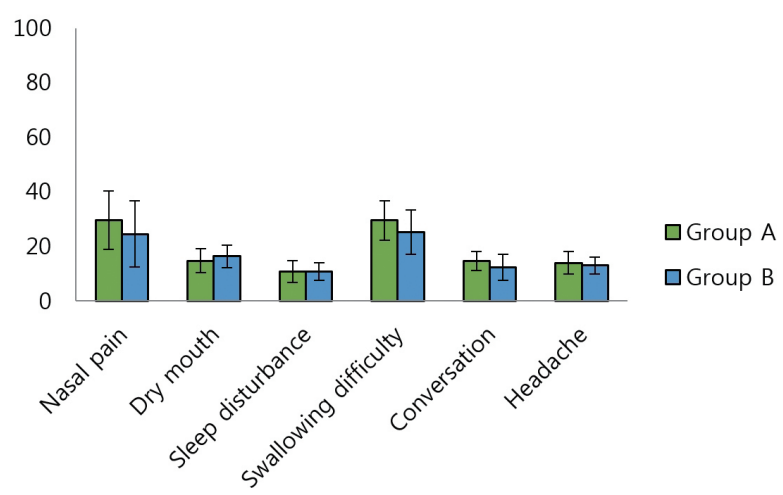

Fig. 4. Comparison of VAS scores on the third post operation day. VAS scores for dry mouth reversed to 14.8 in group A and 16.4 in the group B. But VAS scores of each group had no statistically significant differences. VAS, visual analog scale 
So Young Ji et al. Effectiveness of airway silicone splint

Table 3. Comparison of VAS scores on the fifth post operation day

\begin{tabular}{lrrc} 
Variable & Group A $(n=20)$ & Group B $(n=20)$ & $p$-value ${ }^{\text {a }}$ \\
\hline Nasal pain & $12.6 \pm 5.1$ & $22.6 \pm 7.7$ & $<0.001^{*}$ \\
\hline Dry mouth & $11.4 \pm 3.5$ & $15.8 \pm 3.2$ & $<0.001^{*}$ \\
\hline Sleep disturbance & $8.8 \pm 3.5$ & $9.9 \pm 4.8$ & 0.06 \\
\hline Swallowing difficulty & $23.2 \pm 6.5$ & $22.6 \pm 6.2$ & 0.10 \\
\hline Conversation & $11.3 \pm 4.1$ & $12.6 \pm 5.4$ & 0.37 \\
\hline Headache & $11.0 \pm 4.8$ & $13.0 \pm 4.1$ & 0.10 \\
\hline
\end{tabular}

VAS, visual analog scale.

a) Student's $t$-test; ${ }^{*} p$-value $<0.001$.

Fifth postoperation day

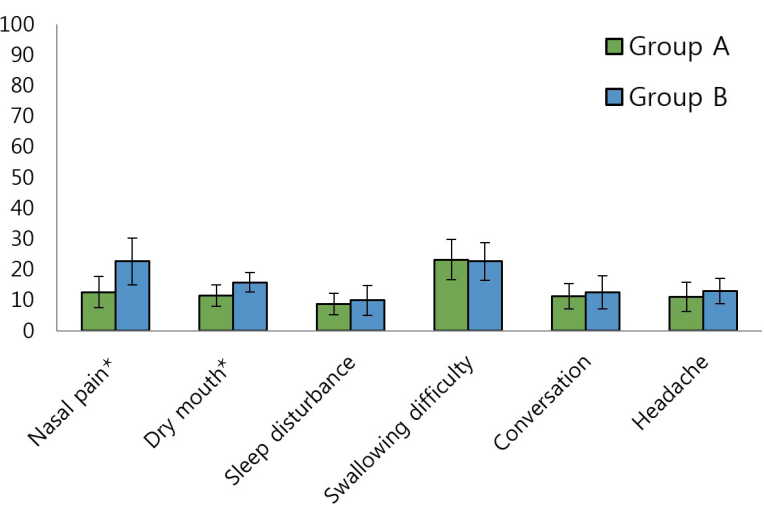

Fig. 5. Comparison of VAS scores on the fifth postoperation day. Group A had statistically significant lower scores for nasal pain, dry mouth than the group B $(P<0.001)$. VAS, visual analog scale. ${ }^{*} p$-value $<0.001$.

in the middle meatus and SPF in the superior meatus.

In the survey of patient discomfort, group B had significantly lower VAS scores for dry mouth, sleep disturbance, conversation difficulty at first postoperative day. Because nasal respiration was possible, there was no need for oral respiration, which improved symptoms, such as dry mouth, sleep disturbance. While group B had lower nasal pain, swallowing difficulty, headache scores, the difference was not statistically significant.

But at third postoperative day most patients of group were able to do nasal breathing, because a good deal of SPF was degraded. So VAS scores of each group had no statistically significant differences.

At fifth postoperative day patients in group A felt more comfortable than group $B$ based on each group's VAS scores. Because nasal irrigation degraded most of SPF during five successive days.

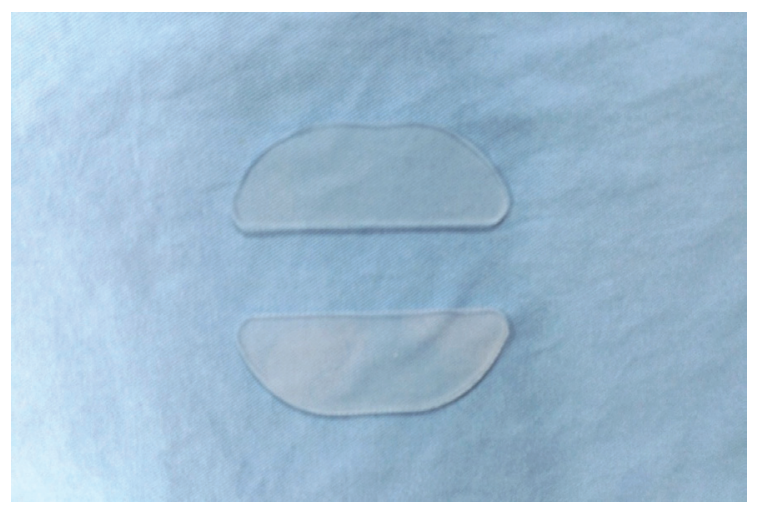

Fig. 6. Silicone sheet type splint (intranasal splint).

Besides NAS had considerable amount of respiration disturbance although it preserved nasal respiration at first postoperative day. And it could trigger foreign body sensation in the nasal cavity. In addition, there was a case of mucosal ulcer in the group $B$ because of continuous mechanical irritation at the trans-septal suture area.

In conclusion, combination method of NAS and SPF have some advantage on patient's comfort from first postoperative day to third postoperative day. Another advantage of the combination method is that it has less pain and bleeding upon packing remov$\mathrm{al}$, and can be useful in providing support after reducing septal fractures. And we suggest use of thin silicone sheet type splint (intranasal splint) (Fig. 6) in patient groups who need more than fourteen days of septal support because of severe septal fracture.

\section{REFERENCES}

1. Moon SH, Baek SO, Jung SN, Seo BF, Lee DC, Kwon H. Efficacy of biodegradable synthetic polyurethane foam for packing nasal bone fractures. JCraniofac Surg 2012;23:1848-50.

2. Yi CR, Kim YJ, Kim H, Nam SH, Choi YW. Comparison study of the use of absorbable and nonabsorbable materials as internal splints after closed reduction for nasal bone fracture. Arch Plast Surg 2014;41:350-4.

3. Kim HY, Kim SR, Park JH, Han YS. The usefulness of nasal packing with vaseline gauze and airway silicone splint after closed reduction of nasal bone fracture. Arch Plast Surg 2012;39:612-7.

4. Choi DS, Lee JW, Yang JD, Chung HY, Cho BC, Choi KY. Minimal packing duration in close reduction for nasal bone fracture treatment. J Plast Surg Hand Surg 2015;49:275-9. 